\title{
A rituximab-based regimen might enable oral steroid avoidance in lupus nephritis
}

$\mathrm{n}$ an observational study, treatment of lupus nephritis (LN) using a rituximabbased therapeutic regimen, termed the 'Rituxilup' protocol, negated the use of oral corticosteroids, without compromising efficacy. Liz Lightstone and colleagues at the Imperial College London NHS Healthcare Trust Lupus Centre, UK, who performed this study claim that it could mark a "step change" in LN therapy.

$\mathrm{LN}$ is a common and severe complication of systemic lupus erythematosus (SLE). Although effective, the standard treatment for LN_comprising oral steroids with either cyclophosphamide or mycophenolate mofetil (MMF) - is not ideal due to the increased morbidity and premature mortality associated with prolonged steroid use. Polypharmacy and cosmetic changes associated with steroids can also result in reduced drug adherence, leading to treatment failure. "We decided that our priority was to minimize steroid use in our patients with SLE, which, combined with our increasing use of rituximab, led us to develop the Rituxilup protocol," explains Lightstone.

The Rituxilup protocol comprises two intravenous doses of $1 \mathrm{~g}$ rituximab with $500 \mathrm{mg}$ methylprednisolone, on days 1 and 15 , and maintenance treatment with MMF. "Since early 2006, this regimen has been used as our standard care in all patients presenting with biopsy proven LN, who are not on maintenance steroids and without life-threatening manifestations," says Lightstone. "We prospectively collected information on these patients to enable us to study a cohort with a suitable follow-up time (at least 1 year, but longer for many)."

The data from the first 50 consecutive patients enrolled reveal that $72 \%$ achieved complete biochemical remission (urine protein:creatinine ratio [PCR] $<50 \mathrm{mg} /$ $\mathrm{mmol}$ ), and an additional $18 \%$ achieved partial remission (PCR $>50 \mathrm{mg} / \mathrm{mmol}$ but $>50 \%$ reduction and non-nephrotic)
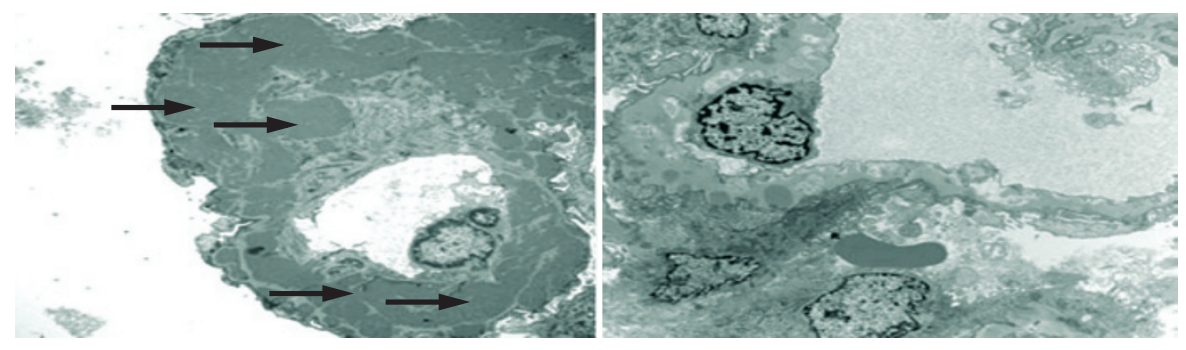

Electromicroscopic images of renal biopsies from a patient with lupus nephritis taken at baseline (left panel) and after 1 year of treatment using the Rituxilup protocol (right panel). The electron-dense dark areas highlighted by arrows indicate glomerular capillary wall deposits at baseline, which are greatly reduced after 1 year of treatment. Courtesy of Liz Lightstone.

by a median of 37 weeks. "Complete remissions were seen early, and the percentage at 1 year was much higher than in the negative LUNAR study, in which rituximab was added on to a background of steroids and MMF," adds Lightstone. Importantly, only two of the 45 responders received $>2$ weeks of oral steroids. In addition, only six systemic SLE flares were recorded. The rate of severe adverse events was low $(n=9)$, which the authors suggest might be due to the absence of oral steroids.

These findings demonstrate that the Rituxilup protocol is safe and efficacious without the requirement for prolonged steroid use. "This is the first time in 60 years that any study has challenged the accepted wisdom that LN has to be treated with oral steroids," claims Lightstone.

"The Rituxilup protocol is clearly effective, as B cells were depleted in almost all patients, $90 \%$ achieved complete or partial remission, very few required oral steroids, and biopsy data showed histological remission even in nine of 15 patients whose clinical response was not ideal," says Anisur Rahman, of University College London, UK, who was not involved in this study. Rahman also agreed with the authors' claim that their paper could mark a "step change" in the treatment of LN, saying "I think they are right, because longterm use of oral steroids in LN has hitherto been universally accepted, and if we could use a regimen that avoids oral steroids altogether it would make a big difference to patients."

Rahman also suggested that the paper raises interesting philosophical questions about how the study was performed. "The authors did not require formal ethics committee approval because they adopted the Rituxilup protocol as their standard care (based on good intellectual reasoning), even though at the time there was no evidence that it was the best treatment," he explains. "Some may argue that it was wrong to adopt the protocol without such evidence, whereas others would argue that this decision was necessary to make progress towards development of a steroidfree regimen for LN and that the end results justify the methods." He concludes, "personally, I tend to the latter view, and support what the authors have done."

Lightstone et al. now have funding for a randomized controlled trial. "We- and we have surveyed the renal and rheumatology community-strongly believe that if the Rituxilup regimen is as effective as MMF plus steroids and has a better safety profile, physicians and patients will switch treatment overnight," opines Lightstone.

\section{David Killock}

Original article Condon, M. B. et al. Prospective observational single-centre cohort study to evaluate the effectiveness of treating lupus nephritis with rituximab and mycophenolate mofetil but no oral steroids. Ann. Rheum. Dis. doi:10.1136/annrheumdis-2012-202844 